\title{
Audit algorithm to increase the competitive stability of a construction company
}

\author{
Angelina Blagodatskaya ${ }^{1, *}$ \\ ${ }^{1}$ Moscow State University of Civil Engineering, 26 Yaroslavskoye shosse, Moscow 129337, Russia
}

\begin{abstract}
The paper presents an algorithm for the implementation of innovative audit when choosing an innovative technology for implementation at the enterprise, which is based on the compliance of the implemented technology with the level of innovative potential of a specific production and economic system. The methodology includes calculating a system of indicators, determining on their basis the level of innovative potential, and then making the optimal decision on the introduction of technology from a bank of possible innovations, which also allows increasing the competitive stability of an enterprise.
\end{abstract}

\section{Introduction}

Nowadays, conditions of competition in the construction market are quite tough. On July 1, 2019, amendments to the Federal Law on Shared-Equity Construction FZ-2014 came into force, which oblige developers to switch from shared-equity construction to project financing using escrow accounts.

Given that the money for the purchased apartment is now deposited into the account after the registration of the contract for participation in shared-equity construction and deposited on it until the registration of ownership of the first apartment in the completed building, the developer must have sufficient working capital to complete the construction of the facility. Small companies are gradually leaving the market, competition in the industry is growing.

Large developers compete among themselves, trying to offer the buyer the most interesting, innovative product. Companies are becoming more flexible, fashionable, relevant, innovative. The construction industry is transforming, moving from the old foundations to active development.

Economic sustainability, financial stability and market stability are components of a company's competitive stability. Scientists previously considered it in terms of these three aspects.

But in the conditions of the modern market, it is impossible not to take into account such an important factor as the presence of innovative potential in absolutely any company. The modern world is designed in such a way that without innovation it is difficult to talk about a reliable future. Innovative potential is a factor that can increase competitive stability even in the face of fierce competition.

\footnotetext{
* Corresponding author: kovalskayaangelina@yandex.ru
} 
Therefore, this paper proposes an algorithm that will increase the level of innovative potential of the enterprise and, as a consequence, its competitive stability.

\section{Experimental part}

The innovative potential of the enterprise (IPE) is part of the resources that have the ability to transform the actual state of the enterprise's production potential into the optimal state defined by the market.

$\underline{I P E}$ reflects the adaptability of $\mathrm{N}$ enterprise for the implementation of specific innovations in the context of the necessary use of the required financial investments, information support, intellectual and human resources. The level of IPE shows how the available resources correspond to the possibilities of introducing the necessary innovations with the further goal of ensuring the competitiveness of the enterprise for a certain period of time.

The components that make up the innovative potential are intellectual resources consisting of industrial property objects, trade secrets and copyright objects. The second necessary component is an innovative infrastructure. And the third component is innovative adaptability [1]. The structure of IPE is shown in Figure 1.

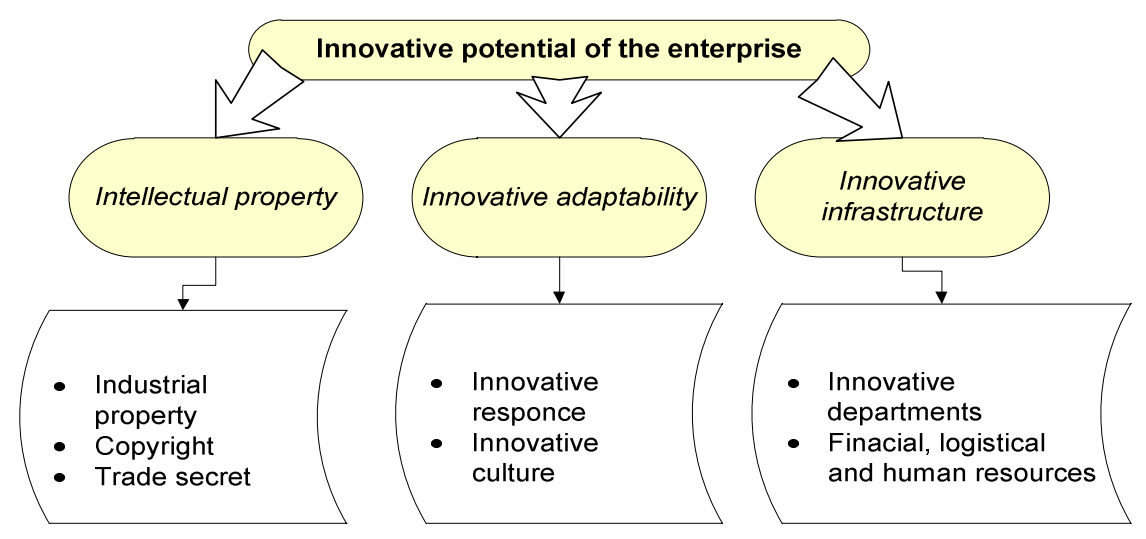

Fig. 1. The structure of the innovative potential of a construction company.

Since it is proposed to include another, fourth component in the structure of competitive stability - the level of IPE, the updated structure of competitive stability will look as follows (Figure 2). 


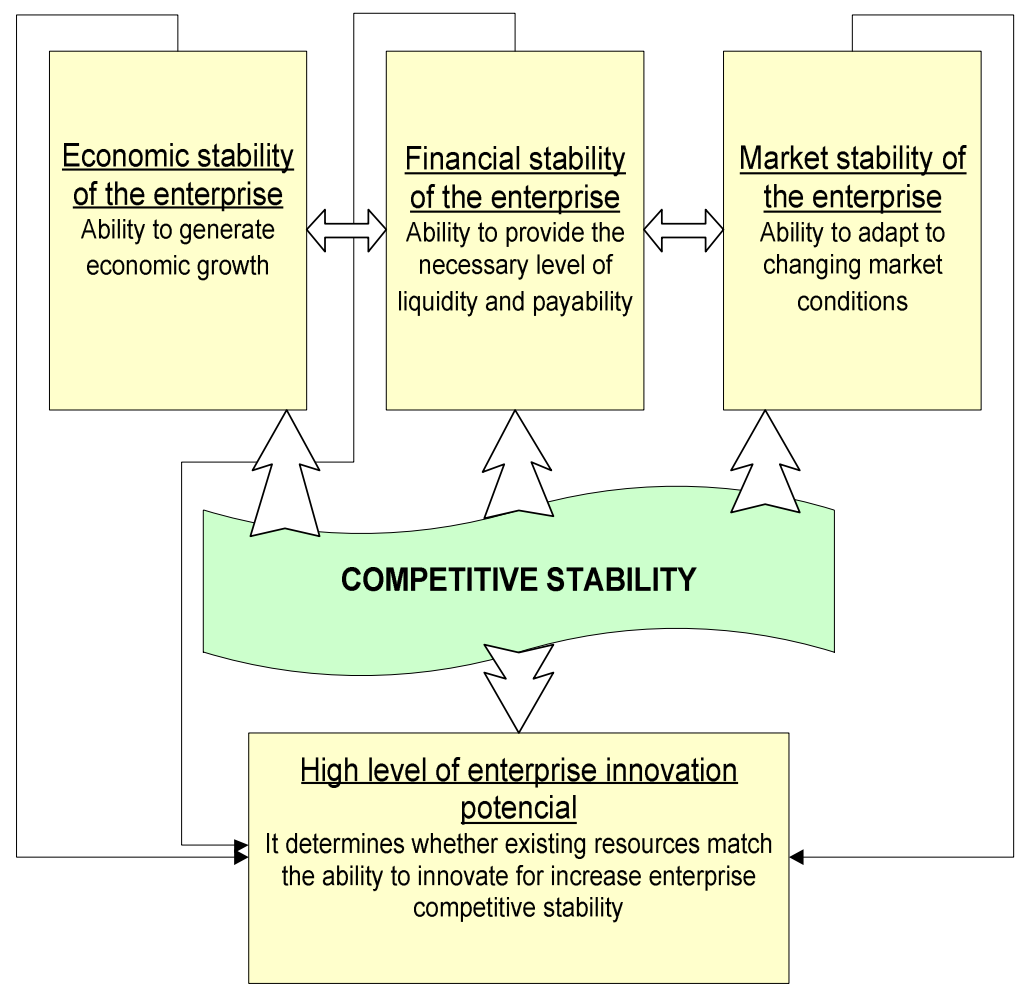

Fig. 2. The structure of the competitive sustainability of a construction company taking into account the innovative component.

A competitively stable position of a company in the market is formed by a number of conditions that take into account the particularities of the theory of innovative development (such as a high level of development of a single information space, transfer of innovations from developed countries, etc.). These conditions are presented in the form of a diagram in Figure 3.

Innovations at a construction company can be implemented in various fields, since construction products have certain specifics. These are such areas as:

-technologies allowing effectively updating the main production assets;

-innovation of management;

-technological innovations to improve the production process;

-innovative building materials. 


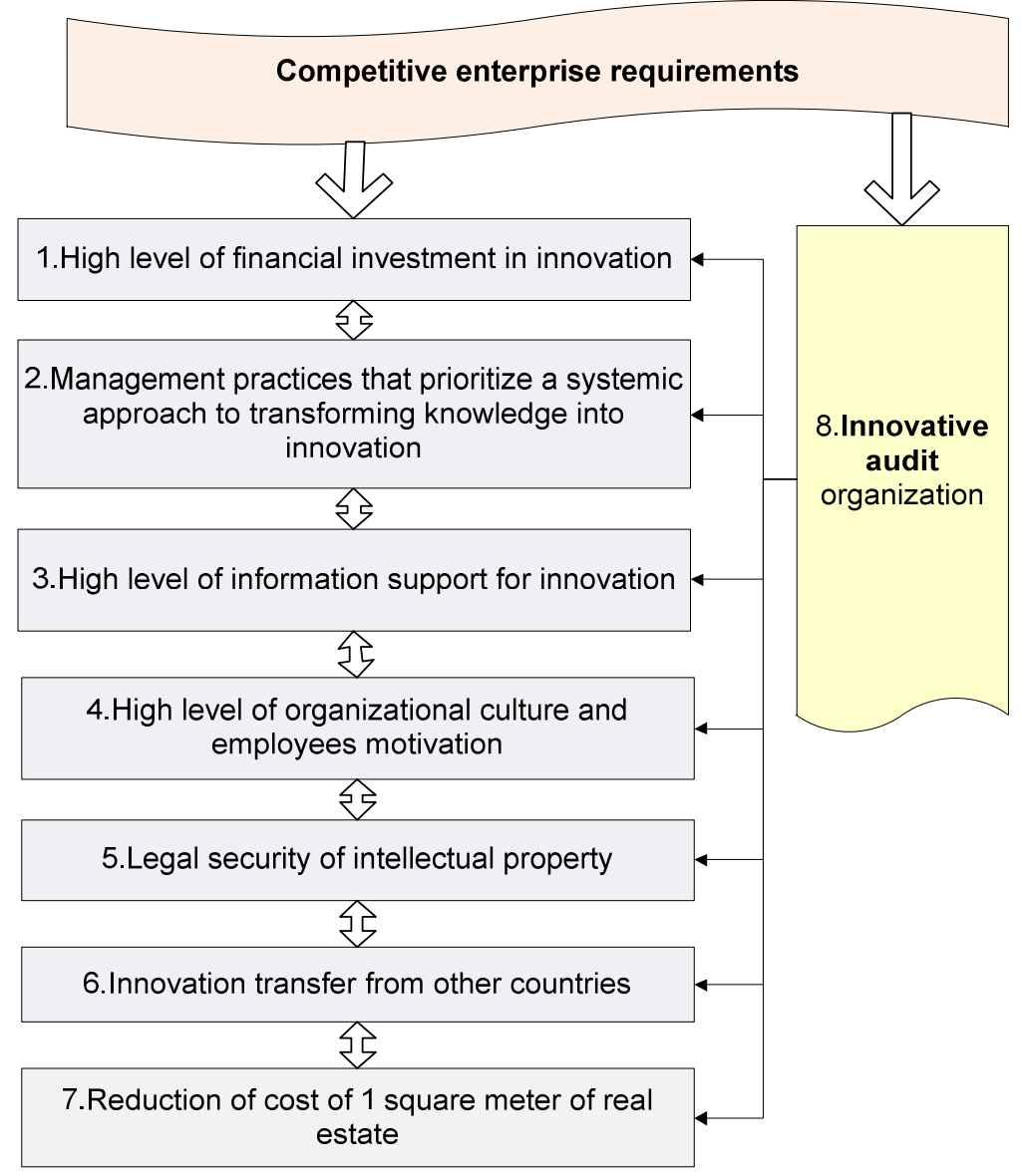

Fig. 3. Conditions for the formation of a competitively stable construction enterprise based on innovative development.

When introducing a specific innovative technology, the effect for the system (in this case, the enterprise) can be both positive and negative. The choice of innovation to increase the level of innovative potential of an enterprise is a process that must take into account all the factors and consequences of introducing technology. In each case, it is possible to use innovative audit [2], [3] to select the optimal innovation.

Innovative audit is an algorithm that allows selecting the best innovative technology for a particular construction company.

\section{Results}

Let's consider the stages of innovative audit.

Stage I. Choice of innovation. We select innovations corresponding to the profile of the company, the choice is made from the innovations available on the market. They will form a bank of possible innovations for the innovation audit process.

Stage II. We select innovations that correspond to the level of innovative potential of this enterprise, taking into account its strategic goal and features. Those innovations that we can actually implement in this enterprise must be selected.

At this stage, it is proposed to rank innovations by cost. This is necessary in order to make an economically feasible decision in the subsequent stages of the audit. 
Stage III. Define the level of IPE of an enterprise. We calculate indicators that characterize innovative potential. There are various methods for assessing the innovative potential of a company. Indicators should reflect:

- Efficiency of investments in innovative activity;

- The amount of R\&D funding and their innovative focus;

- The level of production potential of this company, the condition of all types of resources (human, technological, technical, managerial, informational, intellectual, intangible, legal, financial, environmental);

-Number of employees involved in innovative activity.

To assess the innovative potential of an enterprise, it is proposed to use a methodology according to which the level of innovative potential is determined by the level of intellectual property, the level of innovative adaptability, and the level of innovative infrastructure of an enterprise. To assess the level of intellectual property, a set of indicators is used that characterize industrial property and copyright objects. The level of innovation infrastructure is determined using a set of indicators covering scientific personnel, financial support, material and technical support, and innovative units. The level of innovative adaptability is a complex of indicators of innovative culture, innovative reaction and trade secrets.

In order to compare indicators, we are moving to specific indices. Indices are determined in points by expert assessment (from 0 to 5). A low value of the indicator is determined by points from 0 to 1.5 , the average value is from 1.5 to 3.5 , and the high value is from 3.5 to 5 .

Then, there were determined the intellectual property index $I_{1}$ (as the arithmetic mean of indicators characterizing industrial property and copyright objects), the innovation adaptability index $I_{3}$ (is defined as the arithmetic mean of indicators of innovative culture, innovative reaction and trade secrets) and the innovation infrastructure index $I_{2}$ (is defined as the arithmetic mean of financial indicators support, innovation-oriented units, scientific and managerial personnel, material support).

The integral index of innovative potential (I) is determined by the formula:

$$
\mathrm{I}=\sqrt[3]{k_{1} * I P_{1} \times k_{2} * I P_{2} \times k_{3} * I P_{3}}
$$

Where $k_{1}, k_{2}, k_{3}$ - the coefficients that characterize the weight of factors of intellectual property, innovative infrastructure and innovative adaptability. $k_{1}=0.9 ; k_{2}=0.8 ; k_{3}=0.8$. potential:

After determining the value of the integral index, we determine the level of innovation

- the value of the integral index $0 \leq \mathrm{IP} \leq 1,4$ - low level of innovative potential;

- the value of the integral index $1.4 \leq \mathrm{IP} \leq 2.8$ - average level of innovative potential;

- the value of the integral index $2.8 \leq \mathrm{IP} \leq 4.2$ - high level of innovative potential [4].

After determining the level of innovative potential of a construction company, it is possible to objectively assess the possibility of introducing certain innovations in each case.

Stage IV. Comparison of IPE with innovations selected in stage II. It is necessary to reduce the number of innovations considered for implementation by limiting their number by the level of IPE, which was identified at the previous stage. It is also necessary to consider the economic feasibility of innovation. Cost and possible positive effect must be consistent, this is one of the key factors for choice. Next, we carry out the selection according to two criteria:

1) Innovation should correspond to the level of innovative potential of the company under consideration;

2) Cost.

Stage V. Making the final decision to introduce a specific innovation in the enterprise under consideration. 
Stage VI. Creating a business model. It is further proposed to include the sixth stage in the algorithm for conducting an innovative audit, which will help assessing the effect of introducing the selected specific innovation.

The business model allows describing the basic principles of the creation, development and successful operation of the company. The business model consists of nine structural blocks that need to be completed sequentially, always taking into account the pros and cons of the introduced innovative technology [5].

The innovation audit algorithm is shown in Figure 4.

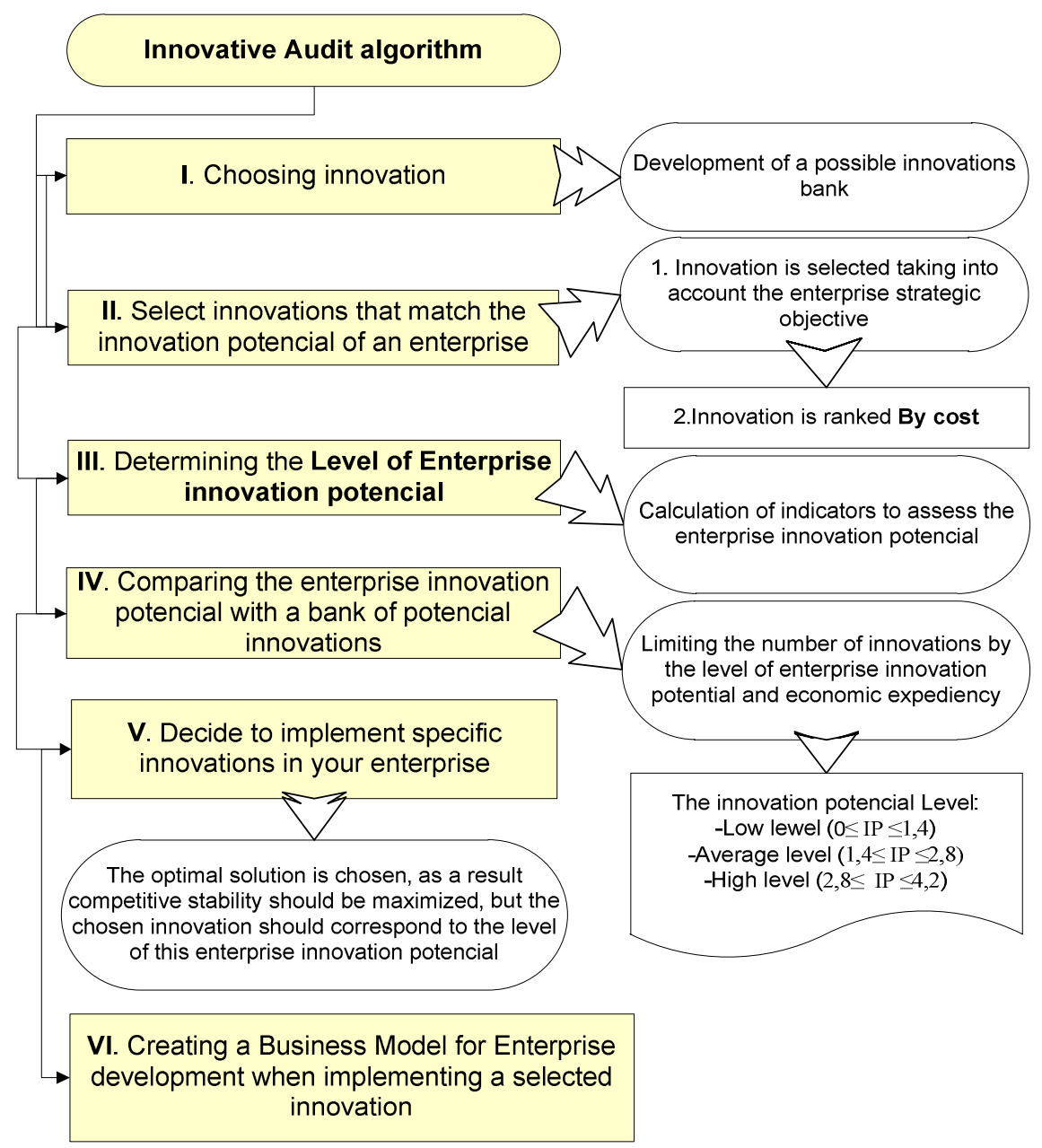

Fig. 4. Algorithm for conducting innovative audit.

\section{Discussions}

The issues of the need to assess the innovative potential of an enterprise and increase its competitive stability were considered in scientific works, periodicals and scientific journals (Kotova E.S. [6], Kolomyts O.N. [7] and others [8-11]).

In their studies, scientists analyze the indicators for assessing innovative potential and note that its level is different for each enterprise, and its increase or decrease is directly related to the competitive stability of the company. However, the authors indicate that, in each case, 
the introduction of innovative technology can give not only a positive but also a negative effect, since the production and economic system may not be ready for this process.

Therefore, there is a need to create an algorithm that will allow selecting the appropriate innovative technology in each case, taking into account the level of innovative potential of an enterprise.

\section{Conclusion}

The proposed algorithm for conducting an innovative audit when choosing an innovative technology allows minimizing risks when introducing innovations for an enterprise, avoiding a negative economic effect, since in the process of conducting an innovative audit, the level of innovative potential of a particular enterprise is assessed. The level of innovation potential shows how susceptible (or not) is the production and economic system to the introduction of new technology. Depending on this level, one innovation is selected from the bank of possible innovations, which will give the system the maximum economic effect, will improve it, since the chosen innovative technology is suitable for a particular enterprise and is compatible with its innovative potential. As a result, the implementation of the successive stages of the innovation audit will make it possible to make an optimal decision on the introduction of innovation, which will subsequently increase the level of competitive stability and ensure reliable functioning of the company in the face of any changes in the market and external economic environment.

\section{References}

1. A.A. Gorobnyak, The estimate method of economic reliability in the development of the urban underground. Procedia Engineering, 165, 1287-1292 (2016)

2. I.A. Doroshenko, S.N. Glagolev, L.A. Minaeva, I.V. Somina, Z.N. Avilova, International Journal of pharmacy and Technology, 8, 4, 22506-22514 (2016)

3. L.Yu. Filobokova, Assessment of the status and use of innovative potential in the strategic audit of small enterprises. Financial management, 27-35 (2017)

4. V.S. Goncharov, Thesis of PhD in Economics. Goncharov Vitaliy Sergeevich. M., p. 185 (2008)

5. A.A. Blagodatskaya, Development of a business model in the process of business planning. Economy and entrepreneurship, 5 (106), 1220-1224 (2019)

6. E.S. Kotova, The main approaches to assessing the innovative potential of the organization. Economic environment (Economicheskaya sreda). 1 (19), 166-171 (2017)

7. O.N. Kolomyts, V.M. Savelenko, Economics and management: problems, solutions, 5, 12, 5-8 (2017)

8. D.V. Valko, I.A. Sergeicheva, Scientific Bulletin of the Volgograd Branch of RANEPA. Series: Economics, No.1, pp. 89-95 (2016)

9. V.V. Bykovsky, Economy. Innovation. Quality control, 3 (12), 81-82 (2015)

10. V. Kankhva, Using the entropy of cover method in the analysis of investment risks. MATEC Web of Conferences 212, $08003 \quad$ (2018) https://doi.org/10.1051/matecconf/201821208003

11. I. Lukmanova, R. Golov, E3S Web of Conferences 33, 02047 (2018) https://doi.org/10.1051/e3sconf/20183302047 